\title{
The Effect Of Fluvoxamine On Sleep Architecture Of Depressed Patients With Insomnia: An 8-Week, Open-Label, Baseline-Controlled Study
}

This article was published in the following Dove Press journal: Nature and Science of Sleep

\author{
Yanli Hao' \\ Yuanyuan $\mathrm{Hu}^{2}$ \\ Haili Wang ${ }^{3}$ \\ Dhirendra Paudel (DD ${ }^{4,5}$ \\ Yan $\mathrm{Xu}^{4-6}$ \\ Bin Zhang ${ }^{4-6}$ \\ 'Department of Human Anatomy, \\ Guangzhou Medical University, \\ Guangzhou 5I I436, People's Republic of \\ China; ${ }^{2}$ Zhongshan Third People's \\ Hospital, Zhongshan, People's Republic of \\ China; ${ }^{3}$ Ganzhou Third People's Hospital, \\ Ganzhou, People's Republic of China; \\ ${ }^{4}$ Department of Psychiatry, Nanfang \\ Hospital, Southern Medical University, \\ Guangzhou, People's Republic of China; \\ ${ }^{5}$ Guangdong-Hong Kong-Macao Greater \\ Bay Area Center for Brain Science and \\ Brain-Inspired Intelligence, Guangzhou, \\ People's Republic of China; ${ }^{6}$ Guangdong \\ Provincial Mental Health Institute, \\ Guangzhou, People's Republic of China
}

Background and aims: Fluvoxamine can markedly increase the serum melatonin level, which regulates human circadian rhythm. However, only limited research has evaluated the effects of fluvoxamine on sleep architecture. Thus, the current study aims to investigate the effect of fluvoxamine on PSG characteristics and the impact of persistent insomnia on the prognosis of depression in the depressed individual with insomnia over the course of 8 weeks.

Methods: Thirty-one clinically depressed patients with insomnia were enrolled in this 8-week, open-label, baseline-controlled study, and 23 patients completed the study. All participants were assigned to receive fluvoxamine for 8 weeks. They were assessed by the PSG, Hamilton Rating Scale for Depression (17 items) (HRSD-17), Clinical Global Index, Pittsburgh Sleep Quality Index, and Epworth Sleepiness Scale at baseline and the following visits, which were at day 14, day 28 , and day 56. A patient with an $\geq 4$ HRSD-17 sleep disturbance factor score at both baseline and endpoint (day 56) was defined as a patient with persistent insomnia.

Results: Compared with baseline, the percentage of stage 3 sleep had significantly $(F=11.630, \mathrm{P}=0.001)$ increased in all 3 visits. Moreover, the percentage of rapid eye movement sleep was reduced during the study, with only a significant difference $(F=3.991$, $\mathrm{P}=0.027)$ between baseline and day 14. Finally, 47.8\% (11/23) of the participants were in remission, and $60.9 \%(14 / 23)$ of them did not report insomnia. The clinical remission ratio of the persistent insomnia group $(11.1 \%[1 / 9])\left(\chi^{2}=8.811, \mathrm{P}=0.004\right)$ was significantly lower than that of the non-insomnia group (71.4\% [10/14]) at the endpoint. Additionally, during the first clinical evaluation (day 14), patients without insomnia had significantly higher final remission ratios than patients with insomnia (80\% [8/10] versus $\left.30.8 \%[4 / 13] ; \chi^{2}=5.79 ; P=0.016\right)$. Conclusion: Fluvoxamine improved PSG parameters and ameliorated complaints of insomnia simultaneously during this 8 -week study. Moreover, depressed individuals who reported persistent insomnia were at higher risk of remaining depressed by the end of the trial, which might be forecasted by the sleep status on day 14 .

Trial registration: The Effect of Fluvoxamine on Polysomnogram in Depressed Patients with Insomnia; https://clinicaltrials.gov/ct2/show/NCT02442713. Registry identifier: NCT02442713. Registry date: May 13, 2015.

Keywords: fluvoxamine, depression, insomnia, polysomnogram

\section{Background Introduction}

For decades, depression has been a major public health issue due to its high prevalence and consequences in the form of disability, morbidity, mortality and
Correspondence: Bin Zhang

Department of Psychiatry, Nanfang Hospital, Southern Medical University, No 1838 , Guangzhou Northern Road, Guangzhou 5I05I5, People's Republic of China

Tel/fax+8620-6278673।

Email zhang73bin@hotmail.com 
socioeconomic burden. The World Health Organization (WHO) predicts that by the year 2020, depression is going to become the second most important cause of disability globally, after cardiovascular disease. ${ }^{1}$ It is noted that $90 \%$ of depressed patients complain about insomnia problems. ${ }^{2,3}$ Furthermore, insomnia adds risks for the new onset of depression and is related to a poor reaction to treatment. ${ }^{4,5}$ Even after effective treatment, the persistence of residual sleep disturbance increases the risk of depression recurrence and suicide. ${ }^{4}$ In addition, the relationship between insomnia and psychiatric conditions is complex with nature of bidirectional causation. ${ }^{6,7}$

According to polysomnography (PSG), which is established as the gold standard for sleep assessment, depression has been associated with several sleep abnormalities: (1) impaired sleep continuity (e.g., prolonged sleep latency (SL), reduced sleep efficiency (SE), and increased awakenings); (2) impaired sleep depth (e.g., decreased stage 3 and increased stage 1); and (3) enhanced rapid eye movement (REM) sleep (e.g., reduced REM latency, increased REM density, and an increased REM sleep during the early half of the night). ${ }^{8,9}$ The first two abnormalities are commonly seen in various psychiatric disorders, while the REM-related abnormalities are characteristic of depression. ${ }^{8}$ These REM-related characteristics might suggest circadian rhythm abnormalities in depression and reinforce the generally accepted phase-advance theory about depression, ${ }^{10}$ which is supported to some extent by the unique insomnia syndrome (early morning waking) in depressive patients. ${ }^{11}$ Thus, it is hypothesized that antidepressants with inherent chronobiotic properties offer a novel way for the treatment of depression. ${ }^{12}$

It is well known that most antidepressants treat depression through 5-hydroxytryptamine (5-HT) neurons, which take an important part in the core behaviours of mood disorders (e.g., mood, appetite, sleep, sexual activity, neuroendocrine functions, motor activity, body temperature, and cognitive function). ${ }^{13}$ 5-HT also affects the sleep-wake cycle regulation, the initiation as well as the maintenance of sleep, and the characteristics of sleep architecture. ${ }^{13}$ Many all-night PSG studies report that tricyclic antidepressants (TCAs) can ameliorate sleep architecture abnormalities in depression by producing rapid suppression of REM sleep, as evidenced by prolongation of REM latency, reduction of REM density, and a decrease in the proportion of REM sleep. ${ }^{14}$ Although TCAs are effective antidepressants, their effects on receptor systems like histaminic, cholinergic, and adrenergic systems, might cause some intolerable adverse effects which limit their clinical usage. ${ }^{15}$ More recently, as a safer treatment alternative, selective serotonin reuptake inhibitors (SSRIs) have been used as first-line drugs for treating the depressive disorder. Compared with TCAs, SSRIs can also suppress REM sleep, and they produce fewer side effects because of their high selectivity for serotonin receptors. ${ }^{16}$ Many short-term $(\leq 4$ weeks $)$ clinical trials suggest that SSRIs often disrupt sleep continuity, while some sub-chronic $(\geq 8$ weeks) clinical trials reported that sleep disturbance diminishes over time. ${ }^{14,17,18}$ This discrepancy might be explained by the accommodative effect, which hypothesizes that homeostatic sleep pressure is enhanced by increasing serotonin release during waking after several weeks of serotoninergic treatment. ${ }^{19}$

Except for the common mechanism of increasing the extracellular serotonin level, each SSRI has unique pharmacological characteristics. For example, accumulating research reports that the melatonin level in serum markedly increased after ingestion of fluvoxamine. ${ }^{20}$ The exact mechanism of increased in melatonin is still unclear, but one probability is that the increase in synthesis of melatonin brought by effects on serotonin, which is a melatonin antecedent. ${ }^{21}$ Another probability is decreased metabolism of melatonin in the liver caused by fluvoxamine. ${ }^{22}$ To date, only three clinical trials have investigated the effects of fluvoxamine on sleep architecture, and their results were contradictory. ${ }^{23-25}$ This discrepancy is possibly due to their different study designs, for example different treatment duration: one was conducted for 12 weeks, ${ }^{23}$ and two were short-term clinical trials $\left(\leq 4\right.$ weeks). ${ }^{24,25}$ In the current study, the effect of fluvoxamine on sleep architecture at different point in time could be elucidated by a series of PSG assessments during the 8-week trial.

\section{Objectives}

The current study aims to investigate the effect of fluvoxamine on PSG characteristics and the impact of persistent insomnia on the prognosis of depression in the depressed individual with insomnia over the course of 8 weeks.

\section{Methods}

\section{Patients And Study Design}

All patients were recruited from three hospitals (Guangdong Provincial Mental Health Centre, Zhongshan The Third People's Hospital, and Ganzhou The Third People's Hospital). The study protocol was examined and 
approved by the Independent Ethics Committees (IEC) from these individual hospitals. Informed consent was taken from the participants in writing. This study was conducted in accordance with the Declaration of Helsinki. Patients were diagnosed with Major depressive disorder (MDD) by psychiatrists using the second version of the Structured Clinical Interview for DSM-IV Axis I Disorders (SCID-II). ${ }^{26}$

The inclusion criteria were:

1. aged 18 to 65 years

2. MDD (Diagnostic and Statistical Manual of Mental Disorders, Fourth Edition, DSM-IV)

3. Moderate to severe depression (Hamilton Rating Scale for Depression (17 items) (HRSD-17) score $\geq 18$ ) and insomnia (sleep disturbance score in HRSD-17 $\geq 4)^{27}$

The exclusion criteria were:

1. any other current or lifetime diagnostic criteria of DSM-IV Axis I mental disorders.

2. a significant sleep disorder (e.g., obstructive sleep apnoea [OSA] and periodic limb movement during sleep [PLMS])

3. a serious medical condition in the last 3 months.

4. significant homicidal or suicidal tendencies (item 3 "suicide" in HRSD-17 $\geq 4$ or medical history)

5. pregnant or breastfeeding

6. night-shift worker

7. having experienced serious adverse events while taking fluvoxamine

After baseline PSG assessment, all patients were arranged to receive fluvoxamine for 8 weeks. At baseline and during the following 3 visits (on day 14, 28 and 56), the participants were assessed using HRSD-17 for clinical improvement. Similarly, sleep quality and daytime sleepiness were evaluated by the Pittsburgh Sleep Quality Index (PSQI) ${ }^{28}$ and the Epworth Sleepiness Scale (ESS), ${ }^{29}$ respectively. And assessment of adverse effects was done using the Treatment Emergent Symptom Scale (TESS-Treatment [TESS-T] and TESS-Severity [TESS-S]). ${ }^{30}$ Fifty milligrams of fluvoxamine were administered at night on the 1 st day. It was then titrated based upon the clinical efficacy and side effects, with a maximum dosage of $300 \mathrm{mg} /$ day. Concomitant use of central nervous system medications during the study, especially hypnotics, was prohibited.

\section{Polysomnographic Study}

At baseline, all participants were assessed by two consecutive nocturnal PSGs. The first-night was considered as an adaptation night, and the PSG variables on the consequent second night were defined as the baseline. Following every 3 visits during the course of the 8-week study, patients were assessed each time by one nocturnal PSG.

According to the night-time PSG, the basic recordings comprised a standard EEG (C4-A1, F4-A1, O2-A1, C3A2), a submental electromyography (EMG), an electrooculography (EOG: LE-A2, RE-A1), an ECG, a bilateral leg EMG, oxyhaemoglobin saturation, nasal airflow pressure, breathing sounds, thoracic and abdominal respiratory efforts, and body position. All sleep variables were categorized into two different groups either sleep continuity indices or sleep architecture indices which were derived using standard criteria from the visual scoring of recordings. Sleep continuity parameters recorded were the total recording time (TRT), total sleep time (TST), SE, SL, REM latency, wake after sleep onset (WASO) and arousal index (AI). Sleep architecture parameters included the percentages of each sleep stage that is REM sleep, stage 1 sleep (S1), stage 2 sleep (S2) and stage 3 sleep (S3) which are proportional part of $\mathrm{TST}^{31}$ The data were edited by technologist specialized in PSG who was blinded to the research study. Sleep stages, periodic limb movements and respiratory events were scored according to AASM criteria at 30-second intervals. ${ }^{31}$ Participants who reported significant PLMS and OSA i.e. periodic limb movement index $(\mathrm{PLMI}) \geq 15$ and apnoea hypopnea $(\mathrm{AHI}) \geq 15$ respectively during the first-night PSG assessment were then removed from the study.

\section{Sample Size}

The estimated sample size for the study was calculated by Statistical \& Power Analysis Software (PASS) using the following solution. In this solution, $\alpha=0.05, \beta=0.1$, the difference of mean value $=2.0$, and the difference of standard deviation=3.0. Although the difference of standard deviation in the previously published literature on SSRI was in the range of $1-2,,^{23-25,32}$ it might be unstable in this study throughout all points (baseline, Day 14, Day 28, and Day 56). So 3.0 should be more reliable. Finally, the number of sample size should be more than 24 patients. We assumed the dropout rate to be $20 \%$, so 30 patients would be enough for this study. 


\section{Recruitment Process}

Sixty-five patients with MDD were initially recruited in this study. Twenty-five patients were further excluded: 17 patients with other DSM-IV comorbid Axis I mental disorders, and 8 patients without insomnia (sleep disturbance score in HRSD-17<4). During the first nocturnal PSG assessment, we excluded 9 patients out of which 4 patients had significant OSA and 5 patients had significant PLMS. Thus, a total of 31 participants with comorbid depression and insomnia entered this study.

During the 8-week study period, 8 patients discontinued the treatment. 5 patients discontinued before Day 14 ( 2 patients due to deterioration of symptoms and combination with other drugs; 2 patients due to the refusal of further sleep tests; and 1 patient due to the emergence of psychotic symptoms that required the addition of antipsychotic drugs). One patient discontinued during Days 14-28 because of refusal to take part in further tests. Two patients discontinued during the Days 28-56 (1 patient due to the emergence of psychotic symptoms that required the addition of antipsychotic drugs and 1 patient refusing to participate in further sleep tests). Ultimately, 23 patients completed this study. The recruitment process is shown in Figure 1.

\section{Data Analysis}

The data were presented as the mean \pm standard deviation for continuous variables and as numbers or percentages for categorical variables. Parametric and non-parametric data were compared using two-sample $t$-tests and MannWhitney $U$-tests, respectively (2 groups). A one-way analysis of variance (ANOVA) and Kruskal-Wallis test were performed for comparing parametric and non-parametric data ( $\geq 3$ groups), respectively. Significant effects in ANOVA were further examined with post hoc tests using the least significant difference method with a Bonferroni correction for multiple comparisons. Mann-Whitney $U$-tests along with adjusted $\mathrm{P}$ values (significant at $\mathrm{P}=0.005$ ) were used for multiple pairwise comparisons. A chi-square test was conducted to test the differences in categorical variables, and multiple chi-squared tests with adjusted $\mathrm{P}$ values (significant at $\mathrm{P}=0.005$ ) were used for multiple pairwise comparisons. A two-sided 5\% level of significance was considered statistically significant. All statistical procedures were conducted using the Statistical Package for the Social Sciences 17.0 for Windows (SPSS, Inc., Chicago, IL).

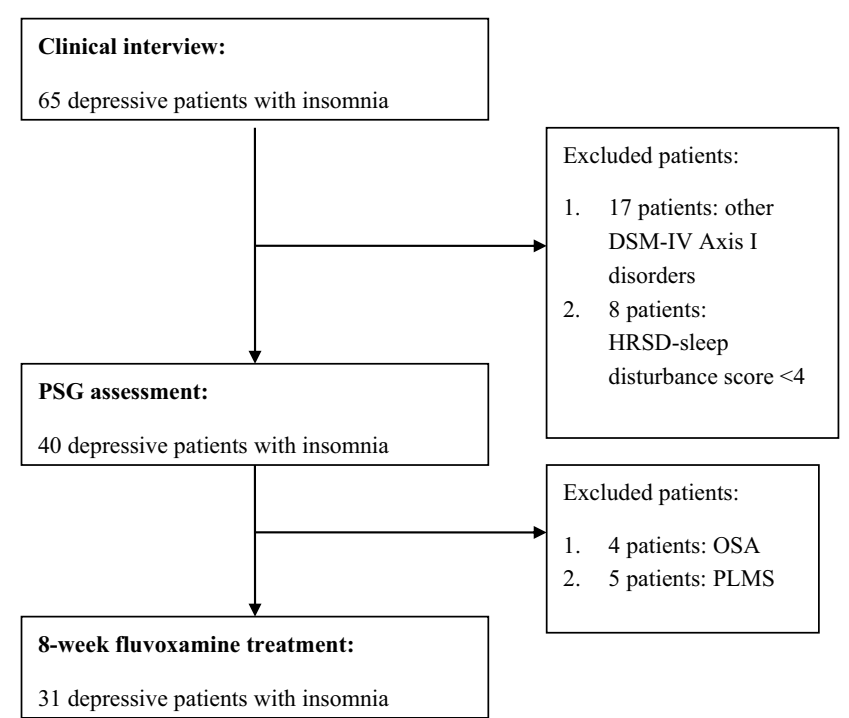

Figure I Flowchart representing the selection of depressive patients with insomnia.

Abbreviations: PSG, polysomnogram; DSM-IV, Diagnostic and Statistical Manual of Mental Disorders Fourth Edition; HRSD, Hamilton Rating Scale for Depression; OSA, Obstructive Sleep Apnoea; PLMS, Periodic Limb Movement during Sleep.

\section{Results}

\section{Demographic And Clinical} Characteristics

All recruited depressed patients were Chinese with Han ethnicity. The participants were predominantly middleaged (mean: 34.1 \pm 8.9 [20-55] years) and female (58.1\%). The demographic and clinical characteristics of this study sample are presented in Table 1 .

\section{Clinical Assessment And Polysomnographic Assessment}

The mean daily doses for fluvoxamine were $133.7 \pm 38.9$ (100-200) mg on Day 14, 144.3 $\pm 61.4(100-300) \mathrm{mg}$ on

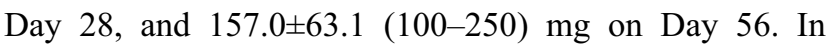
addition, there were only limited side effects (TESS) during the 8-week study. The HRSD-17 score showed improvement on Day 14, and the sleep disturbance factor score in HRSD-17 significantly decreased compared to the score at baseline after Day 28. Over the course of the 8week study, the proportion of remitted participants, as well as participants without insomnia, steadily increased. At the endpoint, $47.8 \%(11 / 23)$ of the patients were in remission, and $60.9 \%(14 / 23)$ of the participants did not report insomnia.

Selected polysomnographic parameters are presented in Table 2. There were no significant differences in the 
Table I Demographic And Clinical Characteristics Of Depressed Patients $(n=31)$

\begin{tabular}{|l|l|}
\hline & $\begin{array}{l}\text { Mean } \pm \text { Standard } \\
\text { Derivation, (Range) Or } \\
\text { Number }\end{array}$ \\
\hline $\begin{array}{l}\text { Demographic characteristics } \\
\text { Age (in years) }\end{array}$ & $34.1 \pm 8.9(20-55)$ \\
Gender (male/female) & $13 / 18$ \\
Marriage (married/single/divorced & $18 / 7 / 6$ \\
or widowed) & \\
Occupation (full-time/part-time/no & $14 / 8 / 9$ \\
job or retired) & $10 / 14 / 7$ \\
Education (university or above/ \\
middle school/primary school or \\
below) \\
Resident (urban/town/rural) & $13 / 12 / 6$ \\
\hline Clinical characteristics & \\
Age onset (in years) & $25.2 \pm 9.1$ (I7-38) \\
Total duration of illness (years) & $8.4 \pm 7.7(0-24)$ \\
Single type/recurrent type & $10 / 21$ \\
Number of illness episodes & $2.8 \pm 1.7$ (I-5) \\
Length of current illness (in weeks) & $5.9 \pm 4.7$ (I-14) \\
\hline
\end{tabular}

TRT during this study. After fluvoxamine treatment, TST and SE became longer and increased in value, respectively, whereas SL and WASO significantly decreased, and REM latency was significantly prolonged. The AI was significantly lower than baseline after Day 28. Moreover, all of the above PSG parameters of sleep continuity shown no significant difference during fluvoxamine treatment. The proportion of S1 decreased during the study and was significantly lower than baseline at Days 28 and 56. The percentage of S2 remained stable during the study. However, the proportion of S3 significantly and gradually increased and was more than $10 \%$ at all 3 visits during fluvoxamine treatment vs. baseline. Compared with baseline, the length of REM sleep shown to decrease during the study, and the difference between baseline and Day 14 was significant.

\section{The Association Between Clinical Performance And The Improvement Of Insomnia}

During the endpoint of this study (Day 56), the insomnia group $(\mathrm{n}=9)$ consumed significantly higher mean daily doses of fluvoxamine (193.5 \pm 70.8 [150-250] $\mathrm{mg}$ vs $127.1 \pm 44.2$ [100-250] $\mathrm{mg}$ ) vs non-insomnia group $(n=14)$. The clinical remission ratio of the non-insomnia group $(71.4 \%[10 / 14])$ was significantly higher in the insomnia group $(11.1 \%[1 / 9]) \quad\left(\chi^{2}=8.811, \mathrm{P}=0.004\right)$. In addition, both groups shared similar and limited side effects. According to the PSG parameters for sleep continuity, both groups shared similar TRT, TST, SE, and REM sleep latency, while the non-insomnia group showed shorter SL and smaller WASO and AI values. According to the PSG parameters for sleep architecture, both groups had a similar percentage of S1 and REM sleep, while the non-insomnia group had a larger percentage of S3 and a smaller percentage of S1 According to self-reports from the two groups, the insomnia group had poor sleep quality (higher PSQI scores); however, both groups showed no similarity in daytime sleepiness (ESS) (Table 3).

Additionally, during the first treatment visit (Day 14), a significant level of insomnia disappeared in $42.3 \%$ (11/26) of the participants. Of the 11 patients without insomnia, 10 patients completed this 8-week study, and 8 patients were defined as being in clinical remission. Of these 15 patients with insomnia on Day 14, 13 patients completed this 8week study, and 4 patients were defined as being in clinical remission. Both groups had significantly different remission ratios $\left(80 \%[8 / 10]\right.$ versus $30.8 \%[4 / 13] ; \chi^{2}=5.79$; $P=0.016)$. Similar to the sleep disturbance factor in HRSD-17, the PSQI and ESS scores were significantly lower compared to baseline after Day 14, and both remained stable during the 8-week trial.

\section{Discussion}

In the present study, it was shown that fluvoxamine was associated with an improvement of depressive symptoms and sleep status (objective PSG parameters and self-report questionnaires) during this 8-week study. Moreover, depressed individuals who reported persistent insomnia were at higher risk of remaining depressed or had failed to achieve a clinically meaningful improvement compared with subjects with non-insomnia.

During the 8 weeks of fluvoxamine treatment, REM latency was significantly prolonged during all visits, and the proportion of REM sleep only significantly decreased on Day 14. Since 1990, a number of researchers have hypothesized that REM suppression was the key therapeutic mechanism of antidepressants. ${ }^{19}$ However, this view was not confirmed because of some inconsistent phenomena. First, Gillin et al administered scopolamine to healthy individuals for 3 days, and these volunteers developed sleep disturbances characteristic of depression, including a shortened REM latency, but they did not develop symptoms of 
Table 2 Clinical And Polysomnographic Parameters Across The Fluvoxamine Treatment In Depressed Patients

\begin{tabular}{|c|c|c|c|c|c|c|}
\hline & $\begin{array}{l}\text { Baseline } \\
(n=3 I)\end{array}$ & $\begin{array}{l}\text { I4th Day } \\
(n=26)\end{array}$ & $\begin{array}{l}\text { 28th Day } \\
(n=25)\end{array}$ & $\begin{array}{l}\text { 56th Day } \\
(n=23)\end{array}$ & Statistics & $\begin{array}{l}\text { Pairwise Comparison } \\
(p<0.05)\end{array}$ \\
\hline Dosage (mg/day) & & $133.7 \pm 38.9$ & $|44.3 \pm 6| .4$ & $157.0 \pm 63.1$ & $F=2.71$ & \\
\hline HRSD & $25.3 \pm 6.8$ & $13.9 \pm 5.6$ & $10.3 \pm 3.5$ & $7.0 \pm 2.3$ & $F=12.54 * * *$ & $a, b, c, e$ \\
\hline $\begin{array}{l}\text { Remitted } \# / \text { nonremitted } \\
\text { (n) }\end{array}$ & $0 / 31$ & $6 / 20$ & $10 / 15$ & $11 / 12$ & $\chi^{2}=19.38^{* * *}$ & $a, b, c$ \\
\hline $\begin{array}{l}\text { HRSD-sleep disturbance } \\
\text { factor }\end{array}$ & $4.3 \pm 3.1$ & $3.6 \pm 2.8$ & $2.6 \pm 1.5$ & $2.4 \pm 1.3$ & $F=6.01 *$ & $b, c$ \\
\hline $\begin{array}{l}\text { Non-insomnia }{ }^{\# \# /} \\
\text { Insomnia (n) }\end{array}$ & $0 / 31$ & $11 / 15$ & $15 / 10$ & $14 / 9$ & $\chi^{2}=29.42^{* * *}$ & a, b, c \\
\hline TESS-S & & $0.8 \pm 0.6$ & $0.7 \pm 0.8$ & $0.7 \pm 0.5$ & $K W=0.69$ & \\
\hline TESS-T & & $0.9 \pm 0.8$ & $0.6 \pm 0.7$ & $0.8 \pm 0.6$ & $K W=0.73$ & \\
\hline PSQI & $15.3 \pm 7.4$ & $9.8 \pm 4.4$ & $6.5 \pm 3.1$ & $6.6 \pm 3.8$ & $F=10.77 * * *$ & $a, b, c$ \\
\hline ESS & $7.8 \pm 4.8$ & $5.2 \pm 3.7$ & $3.5 \pm 3.0$ & $4.1 \pm 3.5$ & $F=9.66 * *$ & $a, b, c$ \\
\hline TRT (min) & $499.8 \pm 80.3$ & $487.3 \pm 81.4$ & $502.1 \pm 63.6$ & $496.3 \pm 67.8$ & $F=1.008$ & \\
\hline TST (min) & $339.5 \pm 111.4$ & $422.1 \pm 69.5$ & $435.6 \pm 83.3$ & $429.0 \pm 78.4$ & $F=5.11 * *$ & $a, b, c$ \\
\hline SE (\%) & $67.9 \pm 25.3$ & $86.6 \pm 29.9$ & $86.7 \pm 27.3$ & $86.5 \pm 31.1$ & $F=4.88^{*}$ & $a, b, c$ \\
\hline $\mathrm{SL}(\mathrm{min})$ & $60.3 \pm 33.2$ & $27.6 \pm 17.0$ & $22.3 \pm 12.1$ & $23.4 \pm 13.8$ & $F=9.38 * * *$ & $a, b, c$ \\
\hline REM Latency (min) & $73.6 \pm 37.0$ & $118.9 \pm 69.1$ & $107.7 \pm 70.9$ & $109.1 \pm 71.6$ & $F=7.05^{* * *}$ & $a, b, c$ \\
\hline WASO (min) & $99.9 \pm 43.1$ & $37.6 \pm 16.4$ & $44.2 \pm 21.8$ & $43.9 \pm 19.3$ & $F=5.93 * *$ & $a, b, c$ \\
\hline Arousal Index & $11.3 \pm 6.9$ & $7.8 \pm 5.9$ & $5.9 \pm 3.8$ & $6.1 \pm 4.5$ & $F=3.664^{*}$ & $b, c$ \\
\hline \% SI & $17.4 \pm 4.3$ & $13.3 \pm 5.0$ & $6.7 \pm 2.6$ & $7.5 \pm 4.2$ & $F=5.098^{* *}$ & $b, c$ \\
\hline \% S2 & $56.8 \pm 21.0$ & $58.2 \pm 18.6$ & $57.4 \pm 17.9$ & $58.0 \pm 20.9$ & $F=I .795$ & \\
\hline$\%$ S3 & $4.6 \pm 1.4$ & $12.6 \pm 5.4$ & $16.7 \pm 8.0$ & $17.6 \pm 7.7$ & $F=11.630 * * *$ & $a, b, c, d, e$ \\
\hline \% REM sleep & $21.4 \pm 8.0$ & $15.9 \pm 7.7$ & $17.2 \pm 10.3$ & $16.8 \pm 8.4$ & $F=3.99 I^{*}$ & $\mathrm{a}$ \\
\hline
\end{tabular}

Notes: ${ }^{\#}$ Remitted: HRSD $\leq 7 .{ }^{~}$ Insomnia: sleep disturbance factor in HSRD $\geq 4$. F: analysis of variance; KW: Kruskal-Wallis test. *: $P<0.05 ; * *$ : $P<0.01$; ***: $P<0.00 I$. Pairwise comparison: a, baseline versus 14 th day; b, baseline versus $28^{\text {th }}$ day; c, baseline versus 56 th day; $d$, 14 th day versus 28 th day; e, 14 th day versus $56^{\text {th }}$ day; $\mathrm{f}$, $28^{\text {th }}$ day versus 56th day.

Abbreviations: HRSD, Hamilton rating scale for depression; TESS-S, treatment-emergent symptom scale-severity; TESS-T, treatment-emergent symptom scale-treatment; PSQI, Pittsburgh sleep quality index; ESS, Epworth sleepiness scale; TRT, total recording time; TST, total sleep time; SE, sleep efficiency; SL, sleep latency; WASO, wake after sleep onset; SI, Stage I sleep; S2, Stage 2 sleep; S3, Stage 3 sleep; REM, rapid eye movement.

depression. ${ }^{33}$ Second, some antidepressants, such as trimipramine, bupropion, and nefazodone, were found to not suppress REM sleep despite being effective antidepressants. $^{14,17}$ Third, the REM suppression effects gradually diminish during chronic treatment; specifically, the proportion of REM sleep is markedly reduced during early stages in therapy and gradually returns towards the baseline. ${ }^{19}$ The third phenomenon was consistent with the present study, in which no significant decrease in REM amount was shown after 2 weeks. This might suggest an accommodative effect of pharmacotherapy on sleep architecture, but it does not attenuate the anti-depressive effect. REM suppression was thought to be mediated most likely through the 5-HT1A receptor. A preclinical study showed that the REM-suppressing effect of citalopram was absent in 5-HT1A-knockout mice. ${ }^{34}$ In humans, drugs that are selective 5-HT1A agonists strongly suppress REM. Additionally, temporary reduction of the availability of 5HT by means of rapid tryptophan depletion in participants treated with SSRIs reversed the REM suppression induced by the antidepressants. ${ }^{35}$ Taken together, the above evidence strongly suggests that 5-HT is crucial in REM regulation, but the association between REM suppression and the antidepressive treatment response remains elusive.

As mentioned before, the alerting effect appears to be a classical phenomenon of SSRIs due to their pharmacological mechanism, ${ }^{13}$ which makes sleep initiation more difficult and disrupts sleep continuity. However, these alerting effects were not shown during all clinical visits (Days 14, 28 and 56). One possible reason is the accommodative effect since it is reported that the alerting effect usually diminishes over time. ${ }^{16}$ Moreover, it was noted that the amount of S3 increased steadily and reached $17.6 \%$ at the endpoint of the study, which is in the normal range of a healthy middle-aged person. ${ }^{36}$ Currently, only a few studies have reported changes in the overall amount of S3 during SSRI therapy. One of our previous studies reported that the percentage of S3 increased in sertraline treatment. ${ }^{18}$ Two other studies, one with 
Table 3 Comparison Of Clinical And Polysomnographic Parameters Between The Insomnia Group ( $n=9$ ) And The Non-Insomnia Group ( $n=14)$ At The End Point Of This Trial (the 56th Day)

\begin{tabular}{|c|c|c|c|}
\hline & Insomnia* $(n=9)$ & Non-Insomnia* $(n=14)$ & Statistics \\
\hline \multicolumn{4}{|l|}{ Clinical parameters } \\
\hline Dosage (mg/d) & $193.5 \pm 70.8$ & $|27| \pm 44.2$. & $T=6.39 ; P=0.003$ \\
\hline HRSD & $10.2 \pm 3.4$ & $5.3 \pm 1.8$ & $T=10.84 ; P=0.000$ \\
\hline Remitted $^{\#} /$ nonremitted (n) & $1 / 8$ & $10 / 4$ & $\chi^{2}=8.811 ; P=0.004$ \\
\hline TESS-S & $0.7 \pm 0.8$ & $0.6 \pm 0.6$ & $Z=0.39 ; P=0.654$ \\
\hline TESS-T & $1.0 \pm 0.8$ & $0.8 \pm 0.9$ & $Z=0.27 ; P=0.703$ \\
\hline PSQI & $8.2 \pm 5.5$ & $5.1 \pm 3.1$ & $T=4.45 ; P=0.019$ \\
\hline ESS & $4.3 \pm 3.6$ & $3.8 \pm 2.2$ & $Z=1.78 ; P=0.437$ \\
\hline \multicolumn{4}{|c|}{ Polysomnographic parameters } \\
\hline \multicolumn{4}{|l|}{ Sleep continuity } \\
\hline TRT (min) & $503.7 \pm 89.4$ & $488.4 \pm 65.1$ & $T=0.97 ; P=0.487$ \\
\hline TST (min) & $4 \mid 7.1 \pm 77.7$ & $436.1 \pm 70.8$ & $T=0.61 ; P=0.325$ \\
\hline SE (\%) & $82.8 \pm 37.2$ & $89.3 \pm 35.6$ & $T=1.32 ; P=0.109$ \\
\hline $\mathrm{SL}(\mathrm{min})$ & $28.6 \pm 14.2$ & $19.8 \pm 8.3$ & $T=4.03 ; P=0.036$ \\
\hline REM sleep latency (min) & $1 \mid 4.5 \pm 58.3$ & $107.1 \pm 49.6$ & $T=0.76 ; P=0.505$ \\
\hline WASO (min) & $57.8 \pm 31.7$ & $32.5 \pm 19.3$ & $T=8.30 ; P=0.016$ \\
\hline Arousal Index & $10.1 \pm 6.9$ & $4.7 \pm 2.5$ & $T=6.88 ; P=0.030$ \\
\hline \multicolumn{4}{|l|}{ Sleep architecture } \\
\hline$\% \mathrm{SI}$ & $11.2 \pm 6.0$ & $4.7 \pm 2.5$ & $T=7.14 ; P=0.007$ \\
\hline$\%$ S2 & $59.5 \pm 20.9$ & $56.8 \pm 20.9$ & $T=0.29 ; P=0.675$ \\
\hline$\%$ S3 & $12.9 \pm 8.4$ & $19.7 \pm 10.5$ & $T=4.30 ; P=0.029$ \\
\hline$\%$ REM sleep & $16.3 \pm 7.5$ & $18.8 \pm 11.3$ & $T=1.83 ; P=0.468$ \\
\hline
\end{tabular}

Notes: *Insomnia: sleep disturbance factor score in HRSD $\geq 4$. ${ }^{*}$ Remitted: HRSD $\leq 7$. T: $t$ test; Z: Mann-Whitney U-test.

Abbreviations: HRSD, hamilton rating scale for depression; TESS-S, treatment-emergent symptom scale-severity; TESS-T, treatment-emergent symptom scale-treatment; PSQI, Pittsburgh sleep quality index; ESS, epworth sleepiness scale; TRT, total recording time; TST, total sleep time; SE, sleep efficiency; SL, sleep latency; WASO, wake after sleep onset; SI, Stage I sleep; S2, Stage 2 sleep; S3, Stage 3 sleep; REM, rapid eye movement.

clomipramine and another with sertraline, reported slow EEG activity (a characteristic wave in S3) to be increased after antidepressant treatment. ${ }^{32,37}$ In the current study, these improvements in sleep architecture were also supported by patients' subjective sleep experiences (PSQI, ESS, and sleep disturbance factor in HRSD-17). The evaluation of the subjective sleep effects of antidepressants is very important for clinicians, because there is often an inconsistency between objective and subjective measurements with anti-depressive therapy. ${ }^{37}$ For example, while the PSG parameters show that the SSRIs affect sleep negatively, at least in early treatment, the subjective questionnaires show improvement. This might be the halo effect of anti-depression. Thus, the ability of patients to perceive their objective sleep accurately is less important clinically than their subjective feelings. ${ }^{38}$ In this study, fluvoxamine could improve subjective sleep experience and objective PSG parameters simultaneously, especially during the early treatment period. Except for the accommodative effect, another reason is one of the fluvoxamine's specific properties of raising serum melatonin levels by inhibiting melatonin metabolism, which may benefit abnormal circadian rhythm and sleep disturbances, even depression itself. ${ }^{39}$

For clinicians, the crucial question is to decide whether sleep status can be linked with clinical repercussions. Our 8week trial study might contribute some knowledge about this issue for fluvoxamine. At the endpoint of this study, the participant who reported insomnia had worse PSG parameters (lower percentage of S3 and a higher percentage of S1) than non-insomnia participants. Thus, self-reported insomnia, which can be gathered easily in clinical practices, was related with sleep architecture to some extent. Moreover, these patients with insomnia at the endpoint consumed significantly higher daily doses for fluvoxamine and had a lower remission ratio than patients without insomnia. These findings suggest that persistent insomnia might act as a barrier to achieving remission during the depression episode. It was consistent with most of the previous studies that reported that anti-depressive treatment fails to provide a sufficient remedy for patients' coexisting persistent insomnia. ${ }^{40}$ This was not 
unexpected, since a number of symptoms of insomnia and depression overlap, such as fatigue, irritability, inattention, and cognitive impairment. ${ }^{41}$ Thus, clinicians should pay separate attention towards insomnia in depressive treatment. To date, the treatment for insomnia in patients with psychiatric disorders could include both pharmacological and behavioural interventions. ${ }^{42}$ This study found that around $50 \%$ of the depressed patients can be remitted by fluvoxamine monotherapy and that this could be forecasted by the insomnia on Day 14 during this study to some extent. In other words, on Day 14, patients with insomnia should be intervened with hypnotics or behavioural interventions. It might not only improve patients' insomnia but also increase the remission rate of depression.

Some cautions should be exerted when interpreting the effects mentioned here. First, no placebo-control was involved in this research, so it cannot be concluded whether fluvoxamine is better than other antidepressants in depressive patients with insomnia. Second, a 1-night adaptive PSG assessment might not be sufficient to dismiss the first-night effect.

\section{Conclusion}

This current research indicates that fluvoxamine was associated with improvements in depressive symptoms and sleep status (PSG parameters and self-report questionnaires) during this 8 -week study. It was shown that depressed individuals who reported persistent insomnia were not likely to achieve a clinically improvement at the end of the investigation. Moreover, it might be forecasted by the insomnia on Day 14 .

\section{Abbreviations}

AI, arousal index; ANOVA, analysis of variance; CBTI, cognitive behavioural therapy for insomnia; DSM, Diagnostic and Statistical Manual of Mental Disorders; ESS, Epworth Sleepiness Scale; HRSD; Hamilton Rating Scale for Depression; IEC, Independent Ethics Committees; IIS, Investigator-Initiated Study; MDD, major depressive disorder; OSA, obstructive sleep apnoea; PLMI, periodic limb movement index; PSG, polysomnography; PLMS, periodic limb movement during sleep; PSQI, Pittsburgh Sleep Quality Index; REM, Rapid eye movement; S1, stage 1 sleep; S2, stage 2 sleep; S3, stage 3 sleep; SE, sleep efficiency; SL, sleep latency; SSRI, selective serotonin reuptake inhibitors; TCA, tricyclic antidepressants; TESS, Treatment Emergent Symptom Scale;
TRT, total recording time; WASO, wake after sleep onset; WHO, World Health Organization.

\section{Ethics Approval And Consent To Participate}

The study protocol was approved by the Independent Ethics Committees (IEC) from three hospitals (Guangdong Provincial Mental Health Centre, Zhongshan The Third People's Hospital, and Ganzhou The Third People's Hospital). Written informed consent for participation was obtained from all patients.

\section{Availability Of Data And Materials}

The data and materials for the current study are available from the corresponding author on reasonable request.

\section{Acknowledgment}

We would like to thank all those who were involved in contributing to this work.

\section{Author Contributions}

All authors contributed to data analysis, drafting and revising the article, gave final approval of the version to be published, and agreed to be accountable for all aspects of the work.

\section{Funding}

The work was supported by funding from the InvestigatorInitiated Study (IIS) from Abbott Pharma (Study Code: FLUVD002) to BZ, the Innovation Project Item of Guangdong Education Department (Grant No. 2018A043442), and the Guangzhou Education Department (Grant No. 2019KC106 to YLH).

\section{Disclosure}

The authors report no conflicts of interest in this work.

\section{References}

1. Murray JC, Lopez A. The global burden of disease: a comprehensive assessment of mortality and disability from disease, injuries and risk factors in 1990. Vol. 15; 1996.

2. Prabha S, Gaynes BN, Wisniewski SR, et al. Insomnia in patients with depression: a STAR*D report. CNS Spectr. 2010;15(6):394-406. doi:10.1017/S1092852900029266

3. Franzen PL, Buysse DJ. Sleep disturbances and depression: risk relationships for subsequent depression and therapeutic implications. Dialogues Clin Neurosci. 2008;10(4):473-481.

4. Baglioni C, Battagliese G, Feige B, et al. Insomnia as a predictor of depression: a meta-analytic evaluation of longitudinal epidemiological studies. J Affect Disord. 2011;135(1-3):10-19. doi:10.1016/j.jad.20 11.01 .011 
5. Fang H, Tu S, Sheng J, Shao A. Depression in sleep disturbance: a review on a bidirectional relationship, mechanisms and treatment. J Cell Mol Med. 2019;23(4):2324-2332. doi:10.1111/jcmm.2019.23.issue-4

6. Krystal AD. Psychiatric disorders and sleep. Neurol Clin. 2012;30 (4):1389-1413. doi:10.1016/j.ncl.2012.08.018

7. Li L, Wu C, Gan Y, Qu X, Lu Z. Insomnia and the risk of depression: a meta-analysis of prospective cohort studies. BMC Psychiatry. 2016;16(1):375. doi:10.1186/s12888-016-1075-3

8. Baglioni C, Nanovska S, Regen W, et al. Sleep and mental disorders: a meta-analysis of polysomnographic research. Psychol Bull. 2016;142(9):969-990. doi:10.1037/bul0000053

9. Abad VC, Guilleminault C. Sleep and psychiatry. Dialogues Clin Neurosci. 2005;7(4):291-303.

10. Harvey AG. Sleep and circadian functioning: critical mechanisms in the mood disorders? Annu Rev Clin Psychol. 2011;7(7):297-319. doi:10.1146/annurev-clinpsy-032210-104550

11. Rd RC, Kupfer DJ. Sleep research in affective illness: state of the art circa 1987. Sleep. 1987;10(3):199-215. doi:10.1093/sleep/10.3.199

12. Palagini L, Baglioni C, Ciapparelli A, Gemignani A, Riemann D. REM sleep dysregulation in depression: state of the art. Sleep Med Rev. 2013;17(5):377-390. doi:10.1016/j.smrv.2012.11.001

13. Monti JM. Serotonin control of sleep-wake behavior. Sleep Med Rev. 2011;15(4):269-281. doi:10.1016/j.smrv.2010.11.003

14. Holshoe JM. Antidepressants and sleep: a review. Perspect Psychiatr Care. 2009;45(3):191-197. doi:10.1111/ppc.2009.45.issue-3

15. Ferguson JM. SSRI antidepressant medications: adverse effects and tolerability. Prim Care Companion J Clin Psychiatry. 2001;3(1):2227. doi:10.4088/PCC.v03n0105

16. Portas CM, Bjorvatn B, Ursin R. Serotonin and the sleep/wake cycle: special emphasis on microdialysis studies. Prog Neurobiol. 2000;60 (1):13-35. doi:10.1016/S0301-0082(98)00097-5

17. Wichniak A, Wierzbicka A, Jernajczyk W. Sleep and antidepressant treatment. Curr Pharm Des. 2012;18(36):5802-5817. doi:10.2174/ 138161212803523608

18. Zhang B, Hao YL, Li XL, Ren YZ, Zhou P, Liu WH. An 8-week, open-label study to evaluate the effect of sertraline on the polysomnogram of depressive patients with insomnia. Sleep Biol Rhythms. 2013;11(3):165-175. doi:10.1111/sbr.2013.11.issue-3

19. McCarthy A, Wafford K, Shanks E, Ligocki M, Edgar DM, Dijk DJ. REM sleep homeostasis in the absence of REM sleep: effects of antidepressants. Neuropharmacology. 2016;108:415-425. doi:10.1016/j.neuropharm.2016.04.047

20. Hartter S, Wang X, Weigmann H, et al. Differential effects of fluvoxamine and other antidepressants on the biotransformation of melatonin. J Clin Psychopharmacol. 2001;21(2):167-174. doi:10.1097/ 00004714-200104000-00008

21. Masters A, Pandi-Perumal SR, Seixas A, Girardin J-L, McFarlane SI. Melatonin, the hormone of darkness: from sleep promotion to ebola treatment. Brain Disord Ther. 2014;4(1):1000151.

22. Reiter RJ. Pineal melatonin: cell biology of its synthesis and of its physiological interactions. Endocr Rev. 1991;12(2):151-180. doi:10.1210/edrv-12-2-151

23. Kupfer DJ, Perel JM, Pollock BG, et al. Fluvoxamine versus desipramine: comparative polysomnographic effects. Biol Psychiatry. 1991;29(1):23-40. doi:10.1016/0006-3223(91)90208-4

24. Wilson SJ, Bell C, Coupland NJ, Nutt DJ. Sleep changes during longterm treatment of depression with fluvoxamine-a home-based study. Psychopharmacology (Berl). 2000;149(4):360-365. doi:10.1007/ s002139900362

25. Silvestri R, Pace-Schott EF, Gersh T, Stickgold R, Salzman C, Hobson JA. Effects of fluvoxamine and paroxetine on sleep structure in normal subjects: a home-based Nightcap evaluation during drug administration and withdrawal. J Clin Psychiatry. 2001;62(8):642652. doi:10.4088/JCP.v62n0812

26. First MB, Spitzer RL, Miriam G, Williams JBW. User's Guide for the Structured Clinical Interview for DSM-IV Axis I Disorders: SCID-II Clinician Version. American Psychiatric Press; 1996.

27. Hamilton M. A rating scale for depression. J Neurol Neurosurg Psychiatry. 1960;23:56-62. doi:10.1136/jnnp.23.1.56

28. Buysse DJ, Reynolds CF 3rd, Monk TH, Berman SR, Kupfer DJ. The pittsburgh sleep quality index: a new instrument for psychiatric practice and research. Psychiatry Res. 1989;28(2):193-213. doi:10.1016/0165-1781(89)90047-4

29. Johns MW. Reliability and factor analysis of the epworth sleepiness scale. Sleep. 1992;15(4):376-381. doi:10.1093/sleep/15.4.376

30. Guy W, National Institute of Mental H, Psychopharmacology Research B, Division of Extramural Research P. ECDEU Assessment Manual for Psychopharmacology: 1976. Rockville (MD): National Institute of Mental Health;1976.

31. Iber C, Ancoli-Israel S, AL C, Quan S: The AASM manual for the scoring of sleep and associated events: rules, terminology and technical specifications; 2007.

32. Jindal RD, Friedman ES, Berman SR, Fasiczka AL, Howland RH, Thase ME. Effects of sertraline on sleep architecture in patients with depression. $J$ Clin Psychopharmacol. 2003;23(6):540-548. doi:10.1097/01.jcp.0000095345.32154.9a

33. JC, Sitaram N, Duncan WC. Muscarinic supersensitivity: A possible model for the sleep disturbance of primary depression? Psychiatry Research. 1979;1(1):17-22.

34. Monaca C, Boutrel B, Hen R, Hamon M, Adrien J. 5-HT 1A/1B receptor-mediated effects of the selective serotonin reuptake inhibitor, citalopram, on sleep: studies in 5-HT 1A and 5-HT 1B knockout mice. Neuropsychopharmacology. 2003;28(5):850-856. doi:10.1038/ sj.npp.1300109

35. Moore P, Gillin C, Bhatti T, et al. Rapid tryptophan depletion, sleep electroencephalogram, and mood in men with remitted depression on serotonin reuptake inhibitors. Arch Gen Psychiatry. 1998;55(6):534539. doi:10.1001/archpsyc.55.6.534

36. Ohayon MM, Carskadon MA, Guilleminault C, Vitiello MV. Meta-analysis of quantitative sleep parameters from childhood to old age in healthy individuals: developing normative sleep values across the human lifespan. Sleep. 2004;27(7):1255-1273. doi:10.1093/sleep/27.7.1255

37. Ehlers CL, Havstad JW, Kupfer DJ. Estimation of the time course of slow-wave sleep over the night in depressed patients: effects of clomipramine and clinical response. Biol Psychiatry. 1996;39 (3):171-181. doi:10.1016/0006-3223(95)00139-5

38. Mayers AG, van Hooff JC, Baldwin DS. Quantifying subjective assessment of sleep and life-quality in antidepressant-treated depressed patients. Hum Psychopharmacol. 2003;18(1):21-27. doi:10.1002/(ISSN)1099-1077

39. Zisapel N. New perspectives on the role of melatonin in human sleep, circadian rhythms and their regulation. Br J Pharmacol. 2018;175 (16):3190-3199. doi:10.1111/bph.v175.16

40. Furihata R, Hall M, Stone K, et al. An aggregate measure of sleep health is associated with prevalent and incident clinically significant depression symptoms among community-dwelling older women. Sleep. 2017;40.

41. Benca RM, Buysse DJ. Reconsidering insomnia as a disorder rather than just a symptom in psychiatric practice. $J$ Clin Psychiatry. 2018;79:1. doi:10.4088/JCP.su17023ah3c

42. Schutte-Rodin S, Broch L, Buysse D, Dorsey C, Sateia M. Clinical guideline for the evaluation of chronic insomnia in adults. $J$ Clin Sleep Med. 2008;4(5):487-504. 


\section{Publish your work in this journal}

Nature and Science of Sleep is an international, peer-reviewed, open access journal covering all aspects of sleep science and sleep medicine, including the neurophysiology and functions of sleep, the genetics of sleep, sleep and society, biological rhythms, dreaming, sleep disorders and therapy, and strategies to optimize healthy sleep.
The manuscript management system is completely online and includes a very quick and fair peer-review system, which is all easy to use. Visit http://www.dovepress.com/testimonials.php to read rea quotes from published authors. 